\title{
Experimental study of tensile testing of metallic Materials in engineering mechanics
}

\author{
Hashentuya \\ Tongliao Vocational College, Tongliao, Inner Mongolia, China \\ 18947513430@163.com
}

Key words: engineering mechanics experiment mechanical property stretching

Abstract. Experiment is critically important in engineering mechanics. This paper analyzes the features of metallic materials through tensile tests, and found the mechanical properties of plastic and brittle materials. Tensile test is one of the most basic and important experiment to measure the mechanical properties of materials under normal temperature and static state. This is not only because tensile test is easy to conduct and analyze but also because the technique is relatively mature. What's more, most of the mechanical indicators of materials used in engineering design such as strength, plasticity and elasticity modulus are mainly based on tensile tests.

\section{Aim of experiment}

1. Verify Hooke's law and measure E of low-carbon steel

2. Measure the strength index of low-carbon steel when it is stretched: yield stress, Rel and tensile strength Rm.

3. Draw pictures of low-carbon steel and gray cast iron when they are stretched and compare their mechanical properties and failure modes.

\section{Experimental facilities and instruments}

Universal tester, vernier caliper and extensometer

\section{Experimental principle}

According to the regulations of the current national standard GB/T 228-2002: Tensile Testing Methods of Metallic Materials at Ambient Temperature, the testing is conducted at a temperature of $10^{\circ} \mathrm{C} \sim 35^{\circ} \mathrm{C}$. The sample should be installed in the chuck of the testing machine. The extensometer should be fixed, then start the machine and subject the sample to slowly increasing tension (the speed of stretching should be determined according to the properties of the material and the aim of testing) until it is broken. Then draw the tensile curve of the material with the automatic drawing device of the testing machine (Fig. 1).

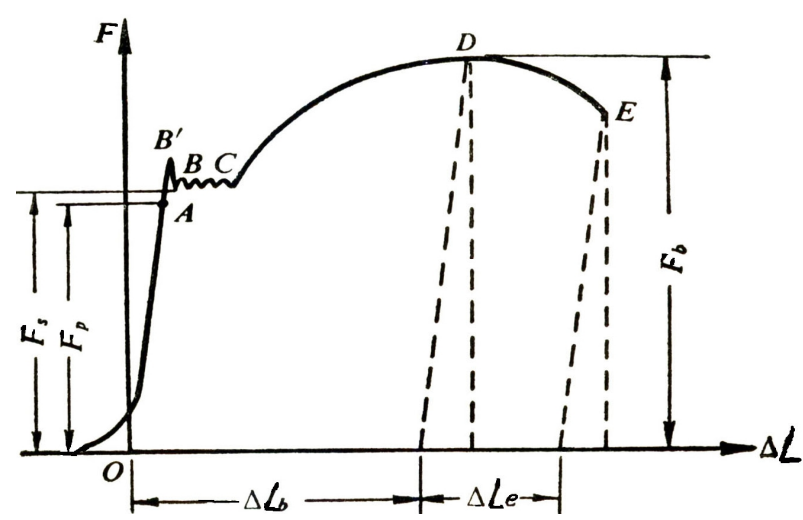

(a) low-carbon steel tensile curve

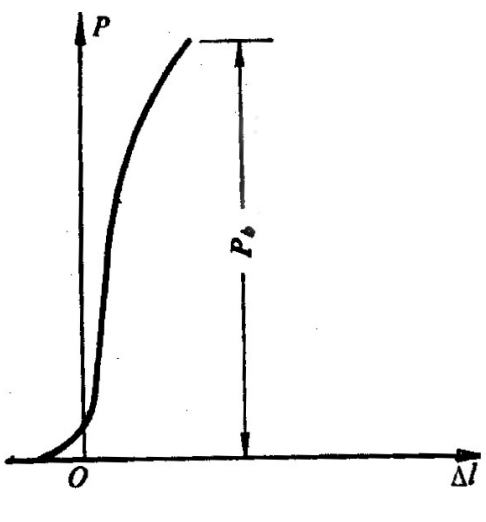

(b) cast iron tensile curve

Fig.1 Tensile curve drawn by the automatic drawing device of the testing machine 


\section{Low-carbon steel (typical plastic material)}

When the tension is relatively weak, the elongation of the sample is in direct proportion to the tensile force, but if the tension exceeds FP, the tensile curve would change from straight to curved. The maximum tension at which a linear relationship is kept is the force value of the proportional limit of the material, FP.

There is a point, Fc, above and nearby FP. If the tension is removed when it is less than Fc, the material will recover its original shape immediately. If the tension is removed when it is above Fc, the shape of the test-piece is only partially recovered. The residual deformation is plastic deformation. So $\mathrm{Fc}$ is the force value stands for elastic limit of materials.

When the tension is added up to a certain degree, the pointer of testing machine which shows force begins to waver or stops and a zigzag or a platform appears on the tensile diagram. This illustrates that the tension the sample receives hardly changes but deformation goes on. This phenomenon is called material yield.

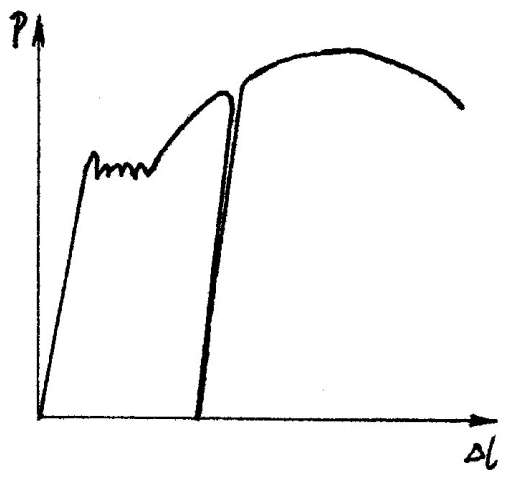

Fig. 2 Cold hardening of low-carbon steel

After the stage of material yield, the force value increases in parallel with deformation and the tension curve goes on rising. This indicates that the material recovers its non-deformability. This phenomenon is called material strengthening. At this stage, the deformation of the sample is mainly plastic deformation which is much greater than the deformation at elastic stage. Before reaching the maximum force, Fm, the deformation within the scope of the sample gauge length is uniform and the tensile curve rises slowly. At this time, it is obvious that the lateral dimension of the sample shrinks. This maximum force, Fm, is the force value of the tensile strength of the material. The tensile strength of a material, Rm, can be obtained through the formula $\mathrm{Rm}=\mathrm{Fm} / \mathrm{S} 0$.

At the strengthening stage of the material, if the tension is reloaded after unloading till the sample snaps, the curve is as Fig. 2. When tension is unloaded, the curve retreats along a line almost parallel to the straight line at the plastic stage instead of retreating along the original stretch curve. This illustrates that before unloading, besides plastic deformation, there is also elastic deformation; if reload after unloading, the curve changes almost along the route of unloading, and then goes on strengthening deformation, as if no unloading ever happened. This phenomenon is called cold hardening. Obviously, cold hardening enhanced the proportional limit and the yield limit of material. However, the plastic properties are reduced in turn.

\section{Cast iron (typical brittle material)}

Brittle materials refer to those materials whose percentage elongation after fracture is $\mathrm{A}<5 \%$. From the time the sample began to be subjected to tension till it snaps, hardly any deformation occurs. What's more, when most brittle materials are stretched, there is no obvious linear segment on the stress-strain curve; there is hardly any plastic deformation and no such phenomena as yield and necking. There is only the stress value at the point of fracture - ultimate strength.

When cast iron sample is subjected to tension and there is still very subtle deformation, the ultimate strength, Fm, would be reached and the sample would snap abruptly. Its strength of 
extension is much weaker than that of low-carbon steel. Similarly, its tensile strength, Rm, can be obtained through the formula $\mathrm{Rm}=\mathrm{Fm} / \mathrm{SO}$; and its percentage elongation after fracture, $\mathrm{A}$, can be got through the formula:

$$
A=\frac{L_{u}-L_{0}}{L_{0}} \times 100 \%
$$

\section{Experimental process}

\section{Tensile experiment of low-carbon steel.}

Our experimental group conducted tensile testing of low-carbon steel on universal tester, and got the tensile diagram shown in Fig. 3.

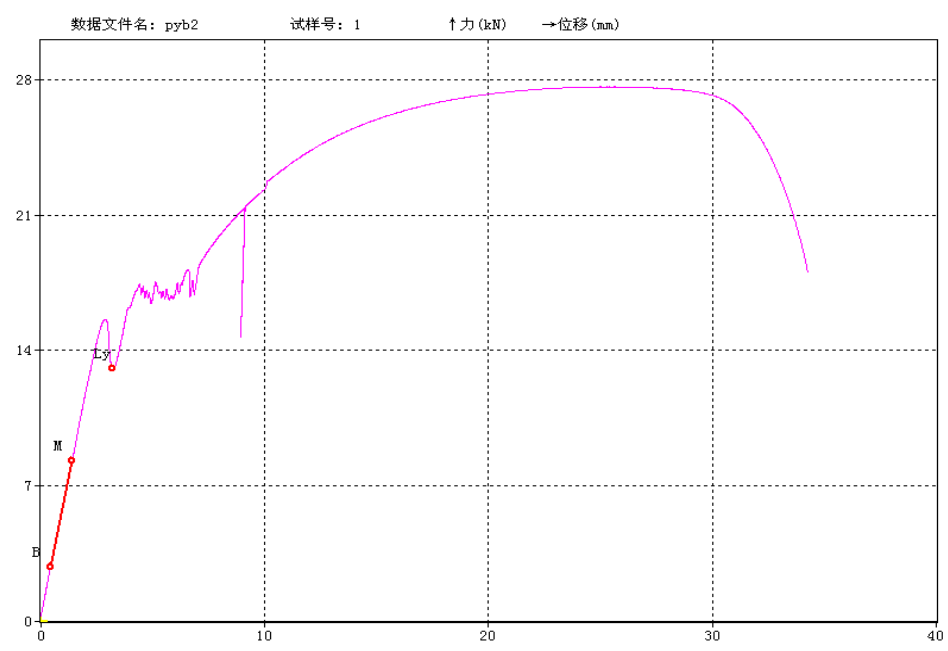

Fig. 3

We can see from the diagram that the experimental result is highly consistent with experimental theories, with obvious elastic stage, yield stage, hardening stage and necking stage. Next, at the hardening stage, there is a vertical line. That's the curve we get after unloading. It verifies cold hardening of material.

Fig. 4 is the fracture shape of low-carbon steel after snapping. We can clearly see the cup-cone shaped fracture.

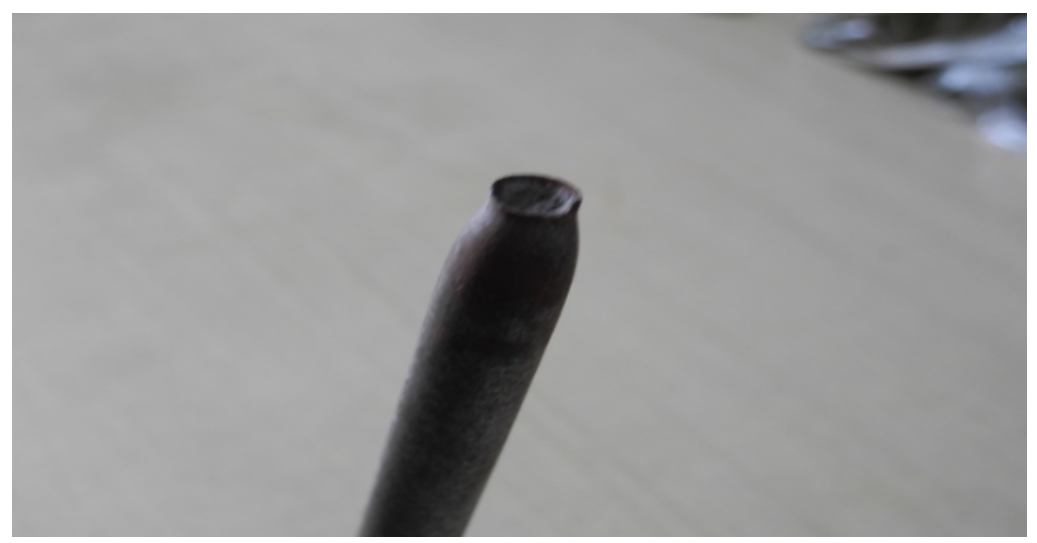

Fig. 4

\section{Tensile experiment of gray cast iron}

After the tensile experiment of low-carbon steel, we also conducted tensile experiment of gray cast iron. The tensile diagram is shown in Fig. 5. 


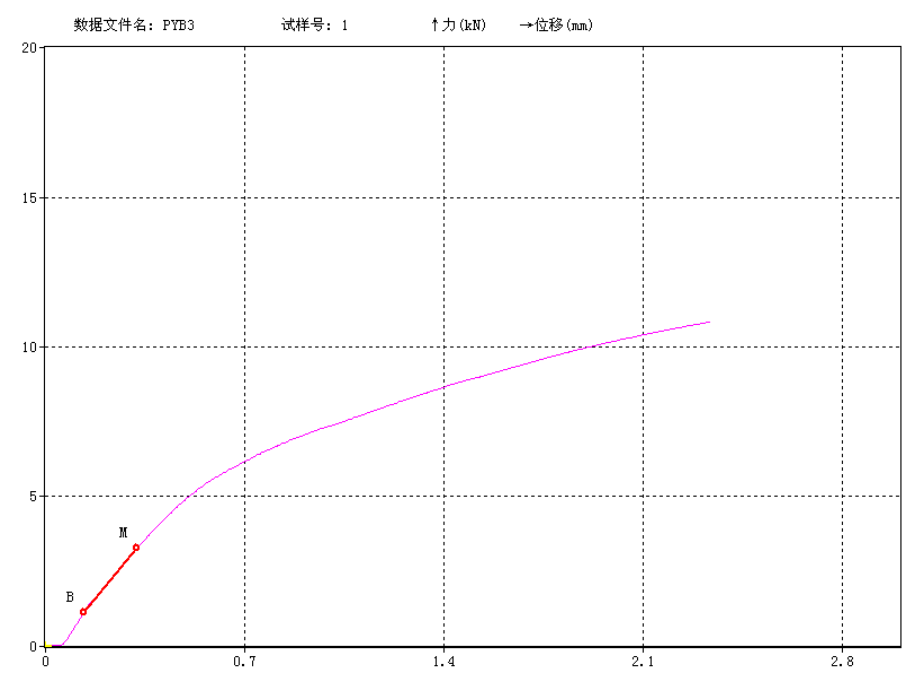

Fig. 5

Similarly, this experimental curve is identical with theoretical curve. This proves that the experiment is very successful. The fracture of gray cast iron is very smooth because it is brittle material and it can be snapped when the stress is not so high.

\section{Conclusion and discussion}

1. Fig. 6 shows the comparison of the samples of low-carbon steel and gray cast iron after fracture. The above conclusion can be seen clearly - the fracture of low-carbon steel is cup-cone shaped and that of gray cast iron is relatively smooth. Meanwhile, we can find that the fracture of gray cast iron is at the junction of transition part and working part, because there is section deformation and stress concentration there. Brittle material is sensitive of stress concentration.

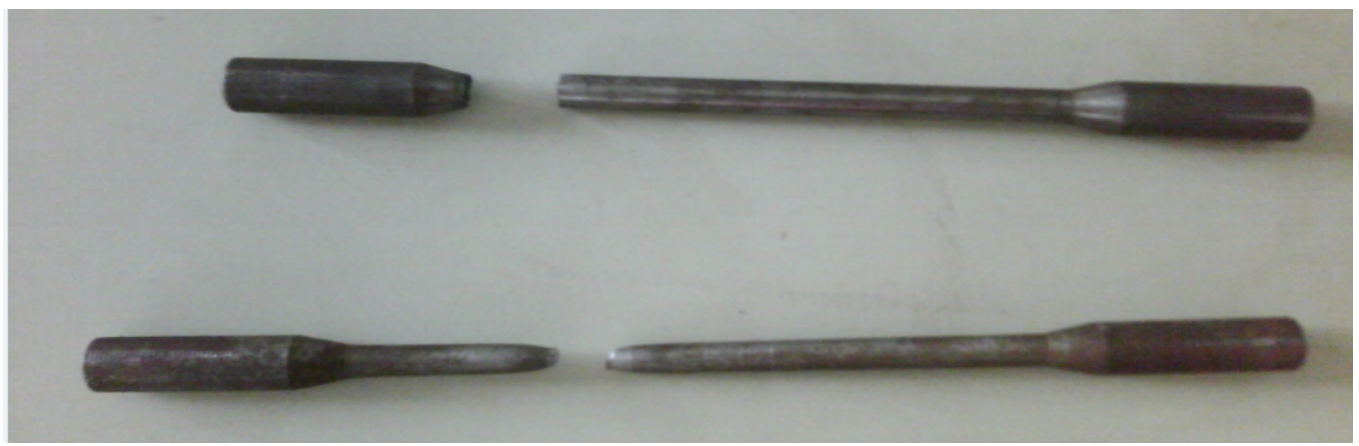

Fig. 6

2. The differences of mechanical properties between low-carbon steel and gray cast iron under normal temperature and static load: low-carbon steel is typical plastic material. Its deformation before fracture is great and it has a high plastic index, so it is highly tensile resistant. Its commonly-used intensity index is its yield limit. Generally speaking, its yield limit value is the same both when it is stretched and compressed.

\section{References:}

[1] Jiang, Ping. The Basis of Engineering Mechanics [M]. Beijing, Higher Education Press, 2003.

[2] Wang, Shouxin. Mechanics of Materials [M]. Dalian, Dalian University of Technology Press, 2014.

[3] Liu, Bin, Yao Yongding \& Ye Guiru. Cable-stayed Bridge Sensor Optimization Research. [J]. Engineering Mechanics, 2005. (05) 